\title{
Application of higher order forms for the description of electromechanical energy converters
}

\author{
TADEUSZ J. SOBCZYK \\ Institute on Electromechanical Energy Conversion, Cracow University of Technology \\ Warszawska 24, 31-155 Kraków, Poland \\ e-mail:pesobczy@cyfronet.pl
}

(Received: 02.07.2010, revised: 01.12.2010)

\begin{abstract}
The purpose of that paper is to develop of unified equations of electromechanical energy converters accounting for the magnetic non-linearity of the main magnetic circuit of a converter. The concept of applying higher order forms of winding currents for the description of the co-energy function is introduced in order to derive the structure of converter equations via mathematical analysis. Also, another concept of equivalent magnetizing currents is applied to determine the higher order forms for selected converters designs. The structure of circuital equations for converters with multiple windings has been unified by means of the introduction of matrices of dynamic and nonlinear inductances following the higher order forms of the co-energy function.
\end{abstract}

Key words: electromechanical converters, co-energy function, higher order forms, magnetizing current, non-linear inductances, dynamic inductances

\section{Introduction}

The so-called Lagrange formalism is a most commonly used way to create equations of electromechanical converters where a converter is modelled in the form of a set of magnetically coupled coils whose locations in space depend on a mechanical variable. In general, the equations of most popular converters with rotary motion can be developed as follows

$$
\begin{gathered}
\frac{\mathrm{d}}{\mathrm{d} t}\left(\frac{\partial E_{\mathrm{mo}}(\mathbf{i}, \varphi)}{\partial i_{n}}\right)+\mathrm{R}_{n} \cdot i_{n}=u_{n}, \quad n \in\{1,2, \ldots, \mathrm{N}\}, \\
\mathrm{J} \frac{\mathrm{d}^{2} \varphi}{\mathrm{d} t^{2}}+\mathrm{D} \frac{\mathrm{d} \varphi}{\mathrm{d} t}=\frac{\partial E_{\mathrm{mo}}(\mathbf{i}, \varphi)}{\partial \varphi}+T_{\mathrm{m}} .
\end{gathered}
$$

Where $E_{\mathrm{mo}}(\mathbf{i}, \varphi)$ refers to the magnetic co-energy (function) stored in the magnetic circuit of a converter which depends on all coil currents constituting the current vector $\mathbf{i}$ and the

\footnotetext{
*This is extended version of a paper which was presented at the 21st Symposium on Electromagnetic Phenomena in Nonlinear Circuits, Essen-Dortmund, 29.06-02.07, 2010.
} 
rotary angle $\varphi$. For $\mathrm{N}$ coils located in a linear magnetic circuit the co-energy function is given by the well-known quadratic form of coil currents

$$
E_{m o}(\mathrm{i}, \varphi)=\frac{1}{2} \sum_{n=1}^{\mathrm{N}} \sum_{k=1}^{\mathrm{N}} \mathrm{L}_{n, k}(\varphi) \cdot i_{n} \cdot i_{k} .
$$

Accounting the converter magnetic circuit saturation makes the co-energy function a multi-variable non-linear function of all involved currents and the rotation angle. The saturation phenomenon has complicated the efforts of many researchers looking for simplified converter models. There exists a number of studies devoted to modelling the saturation effects in electromechanical converters by means of the so-called circuital models. In the second half of the twentieth century a number of researchers have been involved in the development of such models. The most representative analyses can be attributed to [1-4] or [5, 9]. Development of magnetic field computation techniques has changed that trend towards models based on field equations followed by combined circuit-and-field models.

However, the circuital models of electromechanical converters are still very important tools for modelling operational problems in electromechanical systems for the electric energy generations and utilisation. Application of field models or circuit-and-field models for solving such problems seems to be rather controversial. Existing circuital approach to non-linearity is based on two axis generalized models. The approach is not always satisfactory. Therefore, a need for the development of a new type of circuital models have arisen. The purpose of the new models is to represent non-linear phenomena in electromechanical converters in a more precise manner.

The basis for the development of the new models is an approximation of the co-energy function of a converter with a non-linear magnetic circuit. As the co-energy depends on the currents of all windings, it is not easy to find their effective approximation. Having in mind the properties of the co-energy of a set of coils, the co-energy function of electromechanical converters can be approximated by means of a multi-variable power series $[6,8]$ in the following manner

$$
\begin{gathered}
E_{\mathrm{mo}}(\mathbf{i}, \varphi)=\frac{1}{2} \sum_{k=1}^{\mathrm{N}} \sum_{l=1}^{\mathrm{N}} \mathrm{A}_{k, l}(\varphi) \cdot i_{k} \cdot i_{l}+\frac{1}{4} \sum_{k=1}^{\mathrm{N}} \sum_{l=1}^{\mathrm{N}} \sum_{m=1}^{\mathrm{N}} \sum_{n=1}^{\mathrm{N}} \mathrm{A}_{k, l, m, n}(\varphi) \cdot i_{k} \cdot i_{l} \cdot i_{m} \cdot i_{n}+ \\
+\frac{1}{6} \sum_{k=1}^{\mathrm{N}} \sum_{l=1}^{\mathrm{N}} \sum_{m=1}^{\mathrm{N}} \sum_{n=1}^{\mathrm{N}} \sum_{r=1}^{\mathrm{N}} \sum_{s=1}^{\mathrm{N}} \mathrm{A}_{k, l, m, n, r, s}(\varphi) \cdot i_{k} \cdot i_{l} \cdot i_{m} \cdot i_{n} \cdot i_{r} \cdot i_{s}+\cdots
\end{gathered}
$$

The above equation is an equivalent of the Taylor series. The first term of that representtation constitutes a quadratic form of the currents, whereas the following terms are the so-called higher order forms of coil currents, namely, the fourth order form, sixth order form, etc.

To conclude, the purpose of this paper is to reveal fundamentals for the application of the higher order forms in order to create electromechanical converter equations. 


\section{Matrix representation of higher order forms of currents}

It is very useful for many applications to represent a quadratic form in a matrix form

$$
\frac{1}{2} \sum_{n=1}^{\mathrm{N}} \sum_{k=1}^{\mathrm{N}} \mathrm{A}_{n, k}(\varphi) \cdot i_{n} \cdot i_{k}=\frac{1}{2} \mathbf{i}^{T} \cdot \mathbf{A}_{2}(\varphi) \cdot \mathbf{i}
$$

with the constant matrix $\mathbf{A}_{2}(\varphi)$. Analogously, the higher order forms can be written in matrix forms $[6,8]$. The fourth order form can be written as follows

$$
\begin{gathered}
\frac{1}{4} \sum_{k=1}^{\mathrm{N}} \sum_{l=1}^{\mathrm{N}} \sum_{m=1}^{\mathrm{N}} \sum_{n=1}^{\mathrm{N}} \mathrm{A}_{k, l, m, n}(\varphi) \cdot i_{k} \cdot i_{l} \cdot i_{m} \cdot i_{n}=\frac{1}{4} \mathbf{i}^{\mathrm{T}} \cdot \mathbf{A}_{4}(\mathbf{i}, \varphi) \cdot \mathbf{i}= \\
=\frac{1}{4} \mathbf{i}^{\mathrm{T}} \cdot \operatorname{diag}_{\mathrm{N}}\left[\begin{array}{lll}
\mathbf{i}^{T} & \cdots & \mathbf{i}^{T}
\end{array}\right] \cdot \mathbf{A}_{4}^{\mathrm{c}}(\varphi) \cdot \operatorname{diag}_{\mathrm{N}}\left[\begin{array}{lll}
\mathbf{i} & \cdots & \mathbf{i}
\end{array}\right] \cdot \mathbf{i},
\end{gathered}
$$

in which the matrix $\mathbf{A}_{4}^{\mathrm{c}}(\varphi)$ has dimensions $\left(\mathrm{N}^{2} \times \mathrm{N}^{2}\right)$ and its elements are current-independent.

$$
\mathbf{A}_{4}^{\mathrm{c}}(\varphi)=\left[\begin{array}{ccc}
{\left[\begin{array}{ccc}
A_{1111} & \cdots & A_{111 \mathrm{~N}} \\
\vdots & & \vdots \\
A_{11 \mathrm{~N} 1} & \cdots & A_{11 \mathrm{NN}}
\end{array}\right]} & \cdots & {\left[\begin{array}{ccc}
A_{1 \mathrm{~N} 11} & \cdots & A_{1 \mathrm{~N} 1 \mathrm{~N}} \\
\vdots & & \vdots \\
A_{1 \mathrm{NN} 1} & \cdots & A_{1 \mathrm{NNN}}
\end{array}\right]} \\
{\left[\begin{array}{ccc}
A_{\mathrm{N} 111} & \cdots & A_{\mathrm{N} 11 \mathrm{~N}} \\
\vdots & & \vdots \\
A_{\mathrm{N} 1 \mathrm{~N} 1} & \cdots & A_{\mathrm{N} 1 \mathrm{NN}}
\end{array}\right]} & \cdots & {\left[\begin{array}{ccc}
A_{\mathrm{NN} 11} & \cdots & A_{\mathrm{NN} 1 \mathrm{~N}} \\
\vdots & & \vdots \\
A_{\mathrm{NNN} 1} & \cdots & A_{\mathrm{NNNN}}
\end{array}\right]}
\end{array}\right] .
$$

Follow up, the sixth order form can be written as

$$
\begin{gathered}
\frac{1}{6} \sum_{k=1}^{\mathrm{N}} \sum_{l=1}^{\mathrm{N}} \sum_{m=1}^{\mathrm{N}} \sum_{n=1}^{\mathrm{N}} \sum_{r=1}^{\mathrm{N}} \sum_{s=1}^{\mathrm{N}} \mathrm{A}_{k, l, m, n, r, s}(\varphi) \cdot i_{k} \cdot i_{l} \cdot i_{m} \cdot i_{n} \cdot i_{r} \cdot i_{s}=\frac{1}{6} \mathbf{i}^{\mathrm{T}} \cdot \mathbf{A}_{6}(\mathbf{i}, \varphi) \cdot \mathbf{i}= \\
=\frac{1}{6} \mathbf{i}^{\mathrm{T}} \cdot \operatorname{diag}_{\mathrm{N}}\left[\begin{array}{lll}
\mathbf{i}^{T} & \cdots & \mathbf{i}^{T}
\end{array}\right] \cdot \operatorname{diag}_{\mathrm{N}^{2}}\left[\begin{array}{lll}
\mathbf{i}^{T} & \cdots & \mathbf{i}^{T}
\end{array}\right] \cdot \mathbf{A}_{6}^{\mathrm{c}}(\varphi) \times \\
\times \operatorname{diag}_{\mathrm{N}^{2}}\left[\begin{array}{lll}
\mathbf{i} & \cdots & \mathbf{i}
\end{array}\right] \cdot \operatorname{diag}_{\mathrm{N}}\left[\begin{array}{lll}
\mathbf{i} & \cdots & \mathbf{i}
\end{array}\right] \cdot \mathbf{i} .
\end{gathered}
$$

The matrix $\mathbf{A}_{6}^{\mathrm{c}}(\varphi)$, as before, has elements independent on currents, but it has dimensions $\mathrm{N}^{3} \times \mathrm{N}^{3}$. Next higher order forms can be written on the same way.

\section{Matrix forms of converter equations}

By using such matrix notations of the co-energy function (2) can be written in the form

$$
E_{\mathrm{mo}}(\mathbf{i}, \varphi)=\frac{1}{2} \mathbf{i}^{T} \cdot \mathbf{A}_{2}(\varphi) \cdot \mathbf{i}+\frac{1}{4} \mathbf{i}^{T} \cdot \mathbf{A}_{4}(\mathbf{i}, \varphi) \cdot \mathbf{i}+\frac{1}{6} \mathbf{i}^{T} \cdot \mathbf{A}_{\mathbf{6}}(\mathbf{i}, \varphi) \cdot \mathbf{i}+\cdots,
$$

what allows evaluating general Lagrange equations of converters (1) to the form 


$$
\begin{gathered}
\frac{\mathrm{d}}{\mathrm{d} t}(\Psi(\mathbf{i}, \varphi))+\mathbf{R} \cdot \mathbf{i}=\mathbf{u}, \\
\mathrm{J} \frac{\mathrm{d}^{2} \varphi}{\mathrm{d} t^{2}}+\mathrm{D} \frac{\mathrm{d} \varphi}{\mathrm{d} t}=T_{\mathrm{em}}(\mathbf{i}, \varphi)+T_{\mathrm{m}},
\end{gathered}
$$

where:

$$
\Psi(\mathbf{i}, \varphi)=\mathbf{A}_{2}(\varphi) \cdot \mathbf{i}+\mathbf{A}_{\mathbf{4}}(\mathbf{i}, \varphi) \cdot \mathbf{i}+\mathbf{A}_{\mathbf{6}}(\mathbf{i}, \varphi) \cdot \mathbf{i}+\cdots
$$

In electric equations of a converter the matrix of non-linear inductance has been introduced, which has a form

$$
\mathbf{L}_{\mathrm{n}}(\mathbf{i}, \varphi)=\mathbf{A}_{\mathbf{2}}(\varphi)+\mathbf{A}_{\mathbf{4}}(\mathbf{i}, \varphi)+\mathbf{A}_{\mathbf{6}}(\mathbf{i}, \varphi)+\cdots,
$$

than equations (3a) takes the form

$$
\frac{\mathrm{d}}{\mathrm{d} t}\left(\mathbf{L}_{\mathrm{n}}(\mathbf{i}, \varphi) \cdot \mathbf{i}\right)+\mathbf{R} \cdot \mathbf{i}=\mathbf{u} .
$$

The formula for electromagnetic torque takes the form

$$
T_{\mathrm{em}}=\frac{1}{2} \mathbf{i}^{T} \frac{\partial \mathbf{A}_{2}(\varphi)}{\partial \varphi} \mathbf{i}+\frac{1}{4} \mathbf{i}^{\mathrm{T}} \frac{\partial \mathbf{A}_{4}(\mathbf{i}, \varphi)}{\partial \varphi} \mathbf{i}+\frac{1}{6} \mathbf{i}^{\mathrm{T}} \frac{\partial \mathbf{A}_{6}(\mathbf{i}, \varphi)}{\partial \varphi} \mathbf{i}+\cdots
$$

Electric equations can be also written in an alternative form with a matrix of dynamic inductances

$$
\mathbf{L}_{\mathrm{d}}(\mathbf{i}, \varphi) \frac{\mathrm{d} \mathbf{i}}{\mathrm{d} t}+\frac{\mathrm{d} \varphi}{\mathrm{d} t} \frac{\partial \mathbf{L}_{\mathrm{n}}(\mathbf{i}, \varphi)}{\partial \varphi} \cdot \mathbf{i}+\mathbf{R} \cdot \mathbf{i}=\mathbf{u},
$$

where

$$
\mathbf{L}_{\mathrm{d}}(\mathbf{i}, \varphi)=\mathbf{A}_{\mathbf{2}}(\varphi)+3 \cdot \mathbf{A}_{\mathbf{4}}(\varphi, \mathbf{i})+5 \cdot \mathbf{A}_{\mathbf{6}}(\varphi, \mathbf{i})+\cdots
$$

It is rather evident that converter equations can be very easy written in the matrix form using the higher order forms of the co-energy function. The only problem is to find the elements of the matrices $\mathbf{A}_{2}(\varphi), \mathbf{A}_{4}^{\mathrm{c}}(\varphi), \mathbf{A}_{6}^{\mathrm{c}}(\varphi), \ldots$

\section{Determination of higher order forms for converters with sinusoidal MMFs}

Generally, elements of the matrices $\mathbf{A}_{2}(\varphi), \quad \mathbf{A}_{4}^{\mathrm{c}}(\varphi), \quad \mathbf{A}_{6}^{\mathrm{c}}(\varphi), \ldots$ should be found by field computations. For converters with windings generated almost sinusoidal MMFs it is possible to operate with one total MMF, which is determined by its magnitude and angular position. The MMF magnitude is expressed by an equivalent magnetizing current $i_{\mu}[6,8]$, which is given by a quadratic form of currents 


$$
\left(i_{\mu}\right)^{2}=\left(\mathbf{i}^{\prime}\right)^{T} \cdot \mathbf{W} \cdot \mathbf{i}^{\prime}
$$

With a matrix

$$
\mathbf{W}=\left[\begin{array}{cccc}
1 & \cos \left(\mathrm{p} \cdot\left(\alpha_{1}-\alpha_{2}\right)\right) & \cdots & \cos \left(\mathrm{p} \cdot\left(\alpha_{1}-\alpha_{\mathrm{N}}\right)\right) \\
\cos \left(\mathrm{p} \cdot\left(\alpha_{2}-\alpha_{1}\right)\right) & 1 & \cdots & \cos \left(\mathrm{p} \cdot\left(\alpha_{2}-\alpha_{N}\right)\right) \\
\vdots & \vdots & \ddots & \vdots \\
\cos \left(\mathrm{p} \cdot\left(\alpha_{N}-\alpha_{1}\right)\right) & \cos \left(\mathrm{p} \cdot\left(\alpha_{N}-\alpha_{2}\right)\right) & \cdots & 1
\end{array}\right]
$$

An angular position of the MMF $\mathrm{p} \cdot \eta_{\mathrm{p}}$ follows from formula

$$
\tan \left(\mathrm{p} \cdot \eta_{\mathrm{p}}\right)=\sum_{n=1}^{\mathrm{N}} i_{n}^{\prime} \sin \left(\mathrm{p} \cdot \alpha_{n}\right) / \sum_{n=1}^{\mathrm{N}} i_{n}^{\prime} \cos \left(\mathrm{p} \cdot \alpha_{n}\right) .
$$

The co-energy, stored in the main magnetic circuit of a converter, can be expressed as a function of $i_{\mu}$ and $\mathrm{p} \cdot \eta_{\mathrm{p}}$. Then, the co-energy function, when split into two parts: the main magnetic circuit and the leakage zones, can be written as

$$
E_{\mathrm{mo}}(\mathbf{i}, \varphi)=E_{\mu}\left(i_{\mu}, \mathrm{p} \cdot \eta_{\mathrm{p}}\right)+\sum_{n=1}^{\mathrm{N}} E_{\sigma, n}\left(i_{n}\right) .
$$

The above implies the co-energy in the main magnetic circuit depends on a set of parameters describing the total MMF, i.e., the values $i_{\mu}$ and $\mathrm{p} \cdot \eta_{\mathrm{p}}$, whereas the co-energy in the leakage zones depends on the currents of individual windings. Simply, the assumption may be ambiguous, however, it leads to a significant simplification of the description of a converter. The description of the co-energy of an individual winding leakage zone can be considered as a function of currents of windings with the coils in the same slots $E_{\sigma, n}\left(i_{n}, i_{k}\right)$, or as the function $E_{\sigma, n}\left(i_{n}, i_{\mu}, \mathrm{p} \cdot \eta_{\mathrm{p}}\right)$, where the effect of coupling between the main magnetic flux and the leakage flux of an individual winding is expressed.

Detailed formulas for the term $E_{\mu}\left(i_{\mu,}, \mathrm{p} \cdot \eta_{\mathrm{p}}\right)$ depend on the type of a magnetic circuit. In the case of converters with a uniform (smooth) air-gap in which slotting can be neglected, the co-energy can be expressed as a function of the equivalent magnetizing current only, being the even function of that current

$$
E_{\mu}\left(i_{\mu}\right)=\frac{1}{2} \mathrm{C}_{2} \cdot\left(i_{\mu}\right)^{2}+\frac{1}{4} \mathrm{C}_{4} \cdot\left(i_{\mu}\right)^{4}+\cdots
$$

For comparison, in the case of converters with salient-pole rotors that function can be derived as follows

$$
E_{\mu}\left(i_{\mu,}, \mathrm{p} \cdot \eta_{\mathrm{p}}\right)=E_{\mu, 0}\left(\left(i_{\mu}\right)^{2}\right)+E_{\mu, 2}\left(\left(i_{\mu}\right)^{2}\right) \cdot \cos \left(2 \mathrm{p} \cdot \eta_{\mathrm{p}}\right)+E_{\mu, 4}\left(\left(i_{\mu}\right)^{2}\right) \cdot \cos \left(4 \mathrm{p} \cdot \eta_{\mathrm{p}}\right)+\cdots
$$

More detailed considerations on the prediction of the co-energy function in the main magnetic circuit can be found in [7], where, beside of the fundamental $p$-harmonic of the total MMF, the harmonics $5 \mathrm{p}$ or $7 \mathrm{p}$ are taken into account. 
The matrices of higher order forms can be found by expanding the co-energy function (8) and accounting for the formula for the equivalent magnetizing current $i_{\mu}$ and the respective angle $\mathrm{p} \cdot \eta_{\mathrm{p}}$ onto the Taylor series

$$
E_{\mathrm{mo}}(\mathbf{i}, \varphi)=\sum_{k=1}^{\infty} \frac{1}{(2 k) !} \mathrm{d}^{2 k} E_{\mathrm{mo}}(\mathbf{0}, \varphi)
$$

Note that the higher order forms are determined by respective differentials of the co-energy function, i.e., the quadratic form is determined from the second differential, the fourth differential is followed by the fourth order form, etc.

\section{Example of a two-winding converter}

In this section the above mentioned procedure is explained using an example of a converter with two sinusoidal coils, i.e., one coil mounted on a stator and the other coil on a rotor. Moreover, an uniform air-gap is assumed. Limiting the co-energy function to the first two terms leads to

$$
E_{\mathrm{mo}}\left(i_{1}, i_{2}, \varphi\right)=\frac{1}{2} \sum_{k=1}^{2} \sum_{l=1}^{2} \mathrm{~A}_{k, l}(\varphi) \cdot i_{k} \cdot i_{l}+\frac{1}{4} \sum_{k=1}^{2} \sum_{l=1}^{2} \sum_{m=1}^{2} \sum_{n=1}^{2} \mathrm{~A}_{k, l, m, n}(\varphi) \cdot i_{k} \cdot i_{l} \cdot i_{m} \cdot i_{n}
$$

to illustrate the development of the first non-linear term (the four order form). Then, the coenergy function can be written in the matrix form as follows

$$
\begin{aligned}
& E_{\mathrm{mo}}\left(i_{1}, i_{2}^{\prime}, \varphi\right)=\frac{1}{2}\left[\begin{array}{ll}
i_{1} & i^{\prime}{ }_{2}
\end{array}\right]\left[\begin{array}{ll}
\mathrm{A}_{2,1}(\varphi) & \mathrm{A}_{2,2}(\varphi) \\
\mathrm{A}_{2,2}(\varphi) & \mathrm{A}_{2,3}(\varphi)
\end{array}\right]\left[\begin{array}{c}
i_{1} \\
i^{\prime}{ }_{2}
\end{array}\right]+
\end{aligned}
$$

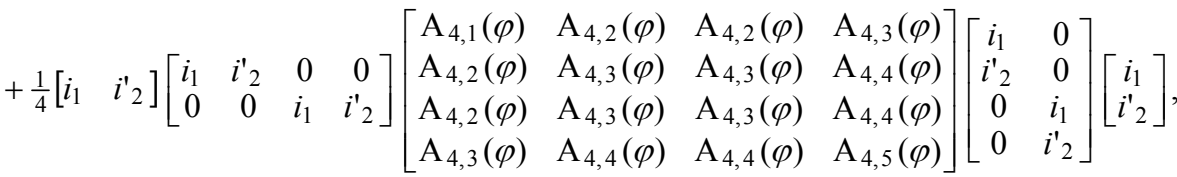

where $i_{2}$ is the current $i_{2}$ recalculated with respect to the winding ' 1 '.

On the other hand, the co-energy function of such converter can be derived as

$$
E_{\mathrm{mo}}\left(i_{1}, i_{2}^{\prime}, \varphi\right)=E_{\mu}\left(\left(i_{\mu}\right)^{2}\right)+E_{\sigma 1}\left(\left(i_{1}\right)^{2}\right)+E_{\sigma 2}\left(\left(i_{2}^{\prime}\right)^{2}\right) .
$$

For converters with an uniform air-gap the co-energy function of the main magnetic circuit is a function of an equivalent magnetizing current only. Therefore, the following relationship occurs

$$
E_{\mu}\left(i_{\mu}\right)=\frac{1}{2} \mathrm{C}_{2} \cdot\left(i_{\mu}\right)^{2}+\frac{1}{4} \mathrm{C}_{4} \cdot\left(i_{\mu}\right)^{4}
$$

The value of the magnetizing current (squared) is given by the quadratic form 


$$
\left(i_{\mu}\right)^{2}=\left[\begin{array}{ll}
i_{1} & i_{2}^{\prime}
\end{array}\right]\left[\begin{array}{cc}
1 & \cos \varphi \\
\cos \varphi & 1
\end{array}\right]\left[\begin{array}{l}
i_{1} \\
i_{2}^{\prime}
\end{array}\right]
$$

The terms representing the leakage zones can be written as follows

$$
E_{\sigma n}\left(\left(i_{n}\right)^{2}\right)=\frac{1}{2} \mathrm{D}_{n, 2} \cdot\left(i_{n}\right)^{2}+\frac{1}{4} \mathrm{D}_{n, 4} \cdot\left(i_{n}\right)^{4} \quad n \in\{1,2\},
$$

assuming that the co-energy depends on own currents only.

Upon evaluation, the constant matrix of the quadratic form can be written as

$$
\mathbf{A}_{2}(\varphi)=\mathrm{C}_{2} \cdot\left[\begin{array}{cc}
1 & \cos \varphi \\
\cos \varphi & 1
\end{array}\right]+\left[\begin{array}{cc}
\mathrm{D}_{2,1} & 0 \\
0 & \mathrm{D}_{2,2}
\end{array}\right]
$$

The constant matrix used for creating the four order form takes the form below

$$
\begin{gathered}
\mathbf{A}_{4}^{\mathrm{c}}(\varphi)=\mathrm{C}_{4} \cdot\left[\begin{array}{cccc}
1 & \cos \varphi & \cos \varphi & \frac{1}{3}(2+\cos 2 \varphi) \\
\cos \varphi & \frac{1}{3}(2+\cos 2 \varphi) & \frac{1}{3}(2+\cos 2 \varphi) & \cos \varphi \\
\cos \varphi & \frac{1}{3}(2+\cos 2 \varphi) & \frac{1}{3}(2+\cos 2 \varphi) & \cos \varphi \\
\frac{1}{3}(2+\cos 2 \varphi) & \cos \varphi & \cos \varphi & 1
\end{array}\right]+ \\
+\left[\begin{array}{cccc}
\mathrm{D}_{4,1} & 0 & 0 & 0 \\
0 & 0 & 0 & 0 \\
0 & 0 & 0 & 0 \\
0 & 0 & 0 & \mathrm{D}_{4,2}
\end{array}\right] .
\end{gathered}
$$

In the same manner the sixth order form can be derived based on the term $\frac{1}{6} \mathrm{C}_{6} \cdot\left(i_{\mu}\right)^{6}$, etc. Therefore, the converter equations take the following form

$$
\frac{\mathrm{d}}{\mathrm{d} t}\left[\begin{array}{cc}
L_{1,1}^{\mathrm{n}} & L_{1,2}^{\mathrm{n}} \\
L_{2,1}^{\mathrm{n}} & L_{2,2}^{\mathrm{n}}
\end{array}\right]\left[\begin{array}{c}
i_{1} \\
i_{2}^{\prime}
\end{array}\right]+\left[\begin{array}{c}
\mathrm{R}_{1} \cdot i_{1} \\
\mathrm{R}_{2}^{\prime} \cdot i_{2}^{\prime}
\end{array}\right]=\left[\begin{array}{c}
u_{1} \\
u_{2}^{\prime}
\end{array}\right],
$$

where the non-linear inductances are determined as follows

$$
\begin{gathered}
{\left[\begin{array}{cc}
L_{1,1}^{\mathrm{n}} & L_{1,2}^{\mathrm{n}} \\
L_{2,1}^{\mathrm{n}} & L_{2,2}^{\mathrm{n}}
\end{array}\right]=\left[\begin{array}{cc}
\mathrm{C}_{2}+\mathrm{D}_{2,1} & \mathrm{C}_{2} \cdot \cos \varphi \\
\mathrm{C}_{2} \cdot \cos \varphi & \mathrm{C}_{2}+\mathrm{D}_{2,2}
\end{array}\right]+\left[\begin{array}{cccc}
i_{1} & i_{2}^{\prime} & 0 & 0 \\
0 & 0 & i_{1} & i_{2}^{\prime}
\end{array}\right] \times} \\
\times\left[\begin{array}{cccc}
\mathrm{C}_{4}+\mathrm{D}_{4,1} & \mathrm{C}_{4} \cdot \cos \varphi & \mathrm{C}_{4} \cdot \cos \varphi & \frac{\mathrm{C}_{4}}{3}(2+\cos 2 \varphi) \\
\mathrm{C}_{4} \cdot \cos \varphi & \frac{\mathrm{C}_{4}}{3}(2+\cos 2 \varphi) & \frac{\mathrm{C}_{4}}{3}(2+\cos 2 \varphi) & \mathrm{C}_{4} \cdot \cos \varphi \\
\mathrm{C}_{4} \cdot \cos \varphi & \frac{\mathrm{C}_{4}}{3}(2+\cos 2 \varphi) & \frac{\mathrm{C}_{4}}{3}(2+\cos 2 \varphi) & \mathrm{C}_{4} \cdot \cos \varphi \\
\frac{\mathrm{C}_{4}}{3}(2+\cos 2 \varphi) & \mathrm{C}_{4} \cdot \cos \varphi & \mathrm{C}_{4} \cdot \cos \varphi & \mathrm{C}_{4}+\mathrm{D}_{4,2}
\end{array}\right]\left[\begin{array}{cc}
i_{1} & 0 \\
i_{2} & 0 \\
0 & i_{1} \\
0 & i_{2}^{\prime}
\end{array}\right] .
\end{gathered}
$$

As such, the expression for the electromagnetic torque in the motion equation below

$$
\mathrm{J} \frac{\mathrm{d}^{2} \varphi}{\mathrm{d} t^{2}}+\mathrm{D} \frac{\mathrm{d} \varphi}{\mathrm{d} t}=T_{\mathrm{em}}\left(i_{1}, i_{2}{ }_{2}, \varphi\right)+T_{\mathrm{m}}
$$


takes the following form

$$
\begin{gathered}
T_{\mathrm{em}}=-\frac{\mathrm{C}_{2}}{2}\left[\begin{array}{ll}
i_{1} & i^{\prime}{ }_{2}
\end{array}\right]^{T}\left[\begin{array}{cc}
0 & \sin \varphi \\
\sin \varphi & 0
\end{array}\right]\left[\begin{array}{c}
i_{1} \\
i^{\prime}{ }_{2}
\end{array}\right]+ \\
-\frac{\mathrm{C}_{4}}{4}\left[\begin{array}{ll}
i_{1} & i_{2}^{\prime}
\end{array}\right]\left[\begin{array}{cccc}
i_{1} & i_{2}^{\prime} & 0 & 0 \\
0 & 0 & i_{1} & i^{\prime}{ }_{2}
\end{array}\right]\left[\begin{array}{cccc}
0 & \sin \varphi & \sin \varphi & \frac{2}{3} \sin 2 \varphi \\
\sin \varphi & \frac{2}{3} \sin 2 \varphi & \frac{2}{3} \sin 2 \varphi & \sin \varphi \\
\sin \varphi & \frac{2}{3} \sin 2 \varphi & \frac{2}{3} \sin 2 \varphi & \sin \varphi \\
\frac{2}{3} \sin 2 \varphi & \sin \varphi & \sin \varphi & 0
\end{array}\right]\left[\begin{array}{cc}
i_{1} & 0 \\
i_{2}^{\prime} & 0 \\
0 & i_{1} \\
0 & i_{2}^{\prime}{ }_{2}
\end{array}\right]\left[\begin{array}{c}
i_{1} \\
i^{\prime}{ }_{2}
\end{array}\right] .
\end{gathered}
$$

Whenever necessary, the matrix of dynamic inductances can be used for the description (5) of the following form

$$
\begin{gathered}
{\left[\begin{array}{cc}
L_{1,1}^{\mathrm{d}} & L_{1,2}^{\mathrm{d}} \\
L_{2,1}^{\mathrm{d}} & L_{2,2}^{\mathrm{d}}
\end{array}\right]=\left[\begin{array}{cc}
\mathrm{C}_{2}+\mathrm{D}_{2,1} & \mathrm{C}_{2} \cdot \cos \varphi \\
\mathrm{C}_{2} \cdot \cos \varphi & \mathrm{C}_{2}+\mathrm{D}_{2,2}
\end{array}\right]+3 \cdot\left[\begin{array}{cccc}
i_{1} & i^{\prime}{ }_{2} & 0 & 0 \\
0 & 0 & i_{1} & i^{\prime}{ }_{2}
\end{array}\right] \times} \\
\times\left[\begin{array}{cccc}
\mathrm{C}_{4}+\mathrm{D}_{4,1} & \mathrm{C}_{4} \cdot \cos \varphi & \mathrm{C}_{4} \cdot \cos \varphi & \frac{\mathrm{C}_{4}}{3}(2+\cos 2 \varphi) \\
\mathrm{C}_{4} \cdot \cos \varphi & \frac{\mathrm{C}_{4}}{3}(2+\cos 2 \varphi) & \frac{\mathrm{C}_{4}}{3}(2+\cos 2 \varphi) & \mathrm{C}_{4} \cdot \cos \varphi \\
\mathrm{C}_{4} \cdot \cos \varphi & \frac{\mathrm{C}_{4}}{3}(2+\cos 2 \varphi) & \frac{\mathrm{C}_{4}}{3}(2+\cos 2 \varphi) & \mathrm{C}_{4} \cdot \cos \varphi \\
\frac{\mathrm{C}_{4}}{3}(2+\cos 2 \varphi) & \mathrm{C}_{4} \cdot \cos \varphi & \mathrm{C}_{4} \cdot \cos \varphi & \mathrm{C}_{4}+\mathrm{D}_{4,2}
\end{array}\right]\left[\begin{array}{cc}
i_{1} & 0 \\
i_{2}^{\prime} & 0 \\
0 & i_{1} \\
0 & i_{2}^{\prime}
\end{array}\right] .
\end{gathered}
$$

It is rather evident that the proposed approach allows one maintain the integrity of the structure of converter equations considering magnetic non-linearity. The approach requires only a few coefficients which can be estimated from field computations or experimental data.

\section{Conclusions}

The paper shows that higher order forms are very effective tool for creation of electromechanical converter equations. They unify description of all important quantities of a converter: co-energy, linked fluxes and matrices of dynamic and nonlinear inductances. Each higher order form is determined by a matrix with constant elements, like a quadratic form is. Elements of that matrix can be found for converters with sinusoidal MMFs using an alternative description of a co-energy by an equivalent magnetizing circuit and an angular position of the total MMF.

\section{References}

[1] Vas P., Generalized analysis of saturated AC machines. Archive für Elektrotechnik 63: 57-62 (1981).

[2] Brown J.E., Kovacs K.P., Vas P., A method of including effects of saturation in the generalized equation of AC machines. IEEE Transactions on PAS 101: 96-103 (1982).

[3] Nehl T.W., Fouad F.A., Demerdash N.A., Determination of saturated values of rotating machinery incremental and apparent inductances by an energy perturbation method. IEEE Transactions on PAS 101: 4441-4451 (1982). 
[4] Vas P., Hallenius K.E., Brown J.E., Cross-saturation in smooth air-gap electrical machines. IEEE Transactions on Energy Conversion 1(1) (1986).

[5] Boldea I., Nasar S.A., Unified treatment of core losses and saturation in the orthogonal-axis model of electrical machines. IEE Proceedings B 134: 355-363 (1987).

[6] Sobczyk T.J., An Energy-Based Approach to Modelling the Magnetic Non-Linearity in AC Machines. Archives of Electrical Engineering, PWN, Warszawa 48 (1999); Part I - General formulas for the co-energy, linked fluxes and inductances. Bull 1-2: 219-229; Part II - General equations of AC machines accounting for saturation due to the main MMF harmonic. Bull 3: 279-294.

[7] Sobczyk T.J., Properties of the co-energy function for AC machines with non-linear magnetic circuit. Periodica Polytechnica, Ser. El. Eng. TU Budapest 45(4) 1-11 (2002).

[8] Sobczyk T.J., Methodological aspects of mathematical modelling of induction machines. (In Polish), WNT Publisher, Warsaw (2004), Chapters 3 \& 5.

[9] El Serafi A.M., Kar N.C., Methods for determining the intermediate-axis saturation characteristics of salient-pole synchronous machines from the measurement d-axis characteristics. IEEE Transactions on Energy Conversion 20(1): 88-97 (2005). 